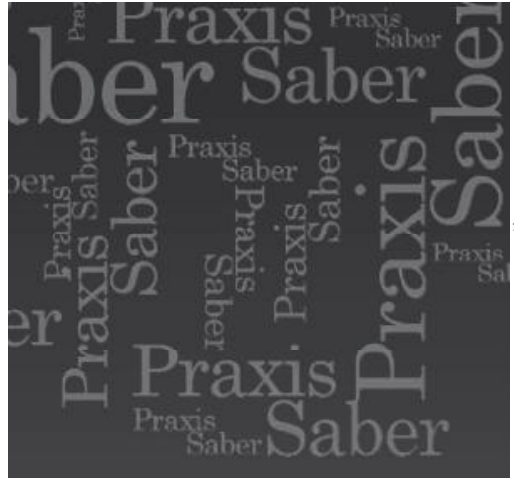

\section{Bruno D'Amore}

Departamento de Matemática, Universidad de Bologna, Italia. bruno.damore@unibo.it

Martha Isabel Fandiño Pinilla Universidad Distrital Francisco José de Caldas, Bogotá, Colombia

Artículo Corto

Recepción: 20 de mayo de 2013

Aprobación: 13 de noviembre de 2013
SOBRE ALGUNAS 'D' EN DIDÁCTICA DE LA MATEMÁTICA: DESIGNACIÓN, DENOTACIÓN, DENOMINACIÓN, DEFINICIÓN, DEMOSTRACIÓN. REFLEXIONES MATEMÁTICAS Y DIDÁCTICAS QUE PUEDEN CONDUCIR LEJOS

\section{Resumen}

En la acción normal del aula, existen momentos delicados a los cuales no se les presta atención por una clara falta de competencia; por ejemplo no se estudian las acciones de designación, de denotación (y connotación), de denominación y de descripción, mientras que las operaciones de definición y de demostración en ocasiones se tienen bajo observación. En este breve texto se quiere llamar la atención sobre estos momentos matemáticos de gran importancia.

Palabras clave: designación, denotación, denominación, definición, demostración. 


\section{About Some 'D' In Didactics of Mathematics: Designation, Denotation, Denomination, Definition, Demonstration. Mathematical and Teaching Reflections That Can go a Long Way.}

\section{Abstract}

In the habitual classroom actions there are delicate moments to which one doesn't pay attention due to a lack of competence; for example, one doesn't pay attention to the actions of designation or denotation (and connotation), denomination and description, while operations of definition and demonstration are much more kept under observation. In this short text, we want to draw attention on all these mathematical moments of great significance.

Key words: designation, denotation, denomination, definition, demonstration.

\section{À Propos De Quelques 'D’ Dans La Didactique Des MathÉMATIQues: Désignation, DÉnOtATION, DÉNOMINATION, DÉFINITION, DÉMONSTRATION. RÉFLEXIONS MATHÉMATIQUES ET DiDACTIQUES QUI Peuvent MENER Loin.}

\section{Résumé}

Lors de l'action normale de salle de classe, il existe des moments délicats au cours desquels l'on ne prête pas attention par un manque évident de 
compétence; par exemple, les actions de désignation, de dénotation (et connotation), de dénomination et de description ne sont pas étudiées, tandis que les opérations de définition et de démonstration sont mises, à l'occasion, sous observation. Le but de ce texte succinct, est d'attirer l'attention sur ces moments mathématiques de grande importance.

Mots clés: désignation, dénotation, dénomination, définition, démonstration.

\section{Sobre Algumas 'D’ Em Didática Da \\ Matemática: Designação, Denotação, DenominaÇÃo, Definição, Demonstração. Reflexões Matemáticas E Didáticas Que Podem Levar Longe}

\section{Resumo}

Nas ações do dia a dia da sala de aula, existem momentos difíceis aos quais não se lhes presta atenção por uma clara falta de competência; por exemplo, não se estudam as ações de designação, de denotação (e conotação), de denominação e de descrição, enquanto as operações de definição e de demonstração às vezes se têm sob observação. Neste breve texto se quer chamar a atenção sobre estes momentos matemáticos de grande importância.

Palavras Chave: designação, denotação, denominação, definição, demonstração. 


\section{Introducción}

La mayor parte de nosotros, seres humanos, confunde entre sí operaciones que los lingüistas y filósofos estudian como no sinónimas; lo hace en la cotidianidad, lo hace en la práctica discursiva, en la comunicación... Y lo hace cuando enseña matemática. Pero dado que siempre nos damos más cuenta de que eventuales fracasos en el aprendizaje de la matemática no son necesariamente vinculados a la falta de comprensión de la disciplina sino a problemas relacionados con el lenguaje, con los diferentes lenguajes, con las acciones semióticas, con los hábitos implícitos etc., que giran alrededor del aprendizaje, consideramos que pudiera tener un cierto interés distinguir y evidenciar las operaciones de designación, de denotación (y connotación), de denominación y de descripción de los objetos matemáticos, ya que cada una de éstas tiene una función precisa. Quisimos también notar que estos términos evocan actividades ligadas a las prácticas del definir y del demostrar, cuyas dificultades de aprendizaje han sido objeto de muchísimas investigaciones específicas.

Sólo como consideración, quisimos evidenciar una vez más cómo este tipo de reflexiones, aunque no muy profundas, muestran que la actitud pragmatista ofrece un mejor marco que la realista para nuestra disciplina. Para esto nos servimos de las reflexiones de John Stuart Mill, economista y filósofo de gran auge en los años sesenta, pero, desafortunadamente, olvidado rápidamente.

\section{Designación, denotación (y connotación), denominación, descripción y definición}

\section{Designación}

Designar tiene varios significados: establecer, estipular o fijar alguna cosa (una fecha, el lugar para una cita, personas a quienes adjudicar algo...); pero también indicar algo con un término apropiado o elegir a alguien para un encargo, proponer un candidato. En su acepción más cercana a la matemática, designar indica una oportuna elección para indicar algo.

Por ejemplo, parece oportuno indicar un segmento con dos letras mayúsculas cada una de las cuales indica sus (puntos) extremos $(A$ y $B$ ) [que algunos escriben $\overline{A B}$, confundiendo el segmento con su medida]; mientras que no sería oportuno ni necesario, aunque no por eso prohibido, 
indicar un segmento con tres letras mayúsculas $(\mathrm{ACB}$, sería únicamente necesario que $C$ pertenezca al segmento $A B \ldots)$.

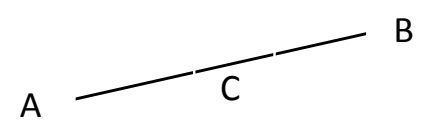

Obviamente, en situaciones particulares, es igualmente oportuno indicar un segmento con una letra minúscula: el segmento $\mathrm{s}$.

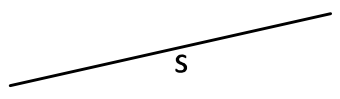

Naturalmente, la designación es un hecho relativo y no absoluto; por ejemplo, en el conjunto de todos los segmentos de un plano dado $\alpha$, no es una designación significativa indicar un segmento dando su longitud (por ejemplo $3 \mathrm{~cm}$.) pues existen infinitos segmentos que corresponden a dicha designación; pero, en un conjunto de segmentos dados, en el cual existe un único segmentos de $3 \mathrm{~cm}$. de largo, aquella designación sería correcta.

La designación no es siempre excelsa, desde un punto de vista matemático; en más de una ocasión se recurre a términos no siempre matemáticamente correctos. Si, por ejemplo, en una misma página aparecen dos cuadrados, uno de ellos algunas líneas más abajo que el otro (tipográficamente), la designación "el cuadrado de arriba", si bien es aceptable en el lenguaje común dado que, precisamente, designa uno de los cuadrados, no es excelsa desde un punto de vista matemático si lo que se desea es hacer referencia a dos cuadrados coplanarios.

La designación tiene muy poca relación con la matemática; designar un objeto matemático es un hecho comunicativo útil para hacer que el emitente y el receptor se entiendan. Sin embargo, en la práctica didáctica el emitente-docente se centra obstinadamente en cuestiones que tienen que ver más con la designación que con aspectos matemáticos, haciendo creer al receptor-estudiante que lo esencial de la matemática está en la designación. Por ejemplo es inútil usar el signo < que aparece a menudo antes de las tres letras mayúsculas con las cuales se designa un ángulo, cuando es precedido de la expresión en palabras "el ángulo"; es decir, escribir: "el ángulo $<A B C$ ", como se lee a veces, es redundante, pedante e inútil. De estas formas de designaciones inútiles se pueden seguir enunciando muchas otras. 


\section{Denotación (y connotación)}

Dado un término, por ejemplo el sustantivo "conejo", la denotación es una frase que lo describe, lo caracteriza, por ejemplo "roedor que..." típica de los diccionarios o de las enciclopedias. No es extraño encontrar los diccionarios en los cuales se denota de forma tal que se crean círculos viciosos:

Circunferencia: curva que circunde el círculo.

Círculo: parte del plano circundada por una circunferencia.

Pero todo término del lenguaje lleva en sí características, por lo general, ligadas más a los sentidos figurativos que al verdadero significado, es decir, significados que acompañan al significado substancial, atributos estilísticos, afectivos, o reenvíos a aspectos literarios (con modalidades positivas o peyorativas o eufemísticas); por ejemplo "conejo" comporta connotativos de falta de coraje o de truhanería. Se habla entonces de connotación.

Por ejemplo, la palabra "recta" tiene una denotación: línea que se puede recorrer sin cambiar dirección (esta no es una definición, sólo es una denotación); pero implica connotaciones que derivan del uso popular y eufemístico: correcto, honrado, honesto, que sigue las reglas etc. $\mathrm{Al}$ estudio de las connotaciones se dedicó el filósofo y economista británico John Stuart Mill (1806 - 1873) quien distingue entre términos (puramente) denotativos (por ejemplo los nombres propios) y términos connotativos (atribuidos a nombres comunes que, además de denotar, connotan). Stuart Mill aplicó esta distinción a su particular concepción de lógica, pensada como verdadero y propio instrumento de pensamiento, productor de ideas.

Distingue aún entre: proposiciones verbales caracterizadas por el hecho de que el predicado expresa un concepto ya contenido en el sujeto (los hombres son racionales: la racionalidad es típica del ser humano y por lo tanto lo denota y lo connota) por tanto, de hecho, privadas de información; proposiciones reales, cuando el predicado expresa un concepto no contenido en el sujeto.

Esto le permite agregar a la lógica, considerada como deductiva (deducción: inferencias que van de lo general a lo particular), o inductiva (inducción: 
inferencias que van de lo particular a lo general), un tercer aspecto que pone como fundamento de los otros, basado sobre la realidad empírica. Para él, en los silogismos la premisa mayor (todos los hombres son mortales) no es más que una expresión general que representa un conjunto de observaciones particulares (cualquier ser humano que evidenciemos, es mortal). Según John Stuart Mill el conocimiento es por tanto un hecho empírico y las generalizaciones son sólo una lista comprimida de casos particulares. También la matemática se incluiría, según este estudioso, en esta acepción: se parte de objetos empíricos sobre los cuales se hacen abstracciones relativas a ciertas propiedades (Mill, 1843).

\section{Denominación}

Se trata de la atribución que se le da a un sustantivo con el fin de identificar o especificar el objeto o la persona o el ente en cuestión. En el análisis lógico propedéutico al latín, se hablaba de complemento de denominación como aquello que introduce una información que especifica algo significativo en referencia al término al cual se refiere. «La ciudad de Bogotá»: "de Bogotá" es el complemento de denominación (en verdad en latín no existía la necesidad de dicho complemento).

\section{Descripción}

La etimología de este término es simple y a la vez problemática: viene del latín "de" que indica cumplimiento de acción y "scribere", escribir. Pero con esta palabra se indica algo complejo que podría expresarse como hacer que el emitente se refiera a un hecho, persona, lugar, y que trace lo más fielmente posible una reproducción en algún sistema descriptivo (por ejemplo semiótico) encaminado a lograr que el receptor pueda hacerse una imagen, una idea, figural o esquemática, o fotográfica que sustituya la original.

En matemática no es usual describir una figura geométrica, por ejemplo, pero se sustituye esta actividad con una imagen visual directa o con una definición. Menos aún se describen otros objetos matemáticos pero no geométricos, como una relación binaria, o una función, o una operación aritmética. Pero la descripción es en ocasiones necesaria pues permite presentar propiedades de los objetos que no pueden ser todos enumerados en una lacónica definición; por ejemplo, en geometría elemental, veamos una descripción del cuadrado: un cuadrado es un rombo que tiene todos 
los ángulos de igual amplitud, las diagonales de igual medida, otros dos ejes de simetría además de las diagonales...

Dicha descripción es en cierto sentido sobreabundante; de hecho, se puede demostrar que si un rombo tiene todos los ángulos de igual medida entonces también las diagonales tienen igual medida o se puede demostrar que tiene otros dos ejes de simetría además de las diagonales y viceversa. La descripción por tanto no es una definición; para definir un cuadrado, basta una sola de estas propiedades, dado que si tomamos una de éstas como hipótesis se deducen como tesis las otras.

\section{Definición}

Consideremos como ejemplo las siguientes dos definiciones: un número natural se dice primo cuando tiene exactamente dos divisores; un cuadrilátero se dice trapecio cuando tiene al menos una pareja de lados paralelos.

Una definición sirve para identificar, para circunscribir, para indicar, para elegir, para designar, para denominar, para denotar, incluso para connotar. Por ejemplo, ¿ 8 es, o no es, un número primo? Veamos cuáles son sus divisores: $1,2,4,8$; pues bien, tiene cuatro divisores, por tanto no es primo. Viceversa, 5 tiene como divisores sólo 1 y 5 , exactamente dos, por tanto 5 es un número primo.

Podríamos preguntarnos, ¿y si en lugar de definir los enumeramos todos? Cierto, es una solución; entonces los números primos son aquellos que aparecen en la siguiente lista: 2, 3, 5, 7, 11,13... Bien, ¿pero qué encontramos después de la coma? Por ejemplo ¿177778905647344442109707 está, o no, en la lista? La dificultad de dar una respuesta a esta pregunta muestra cómo lo compacto de la definición proporciona un instrumento, donde una lista completa es imposible (como en el caso de los números primos que son notoriamente infinitos) o demasiado compleja cuando la lista no se puede proponer (¿Carlos Eduardo Vasco Uribe es mayor de edad? ¿Entra en la lista mundial completa de todas las personas mayores de edad? ¿Quién podría concretar una lista de este tipo? Mucho mejor sería pedirle si ya cumplió 18 años, aprovechando la definición).

El uso de definiciones, como hemos visto, no es peculiar de la matemática; tomemos un ejemplo de la vida de todos los días al cual hicimos alusión 
líneas arriba: mayor de edad es todo ser humano que ya cumplió 18 años. El mismo discurso hecho para la lista de los mayores de edad vale para el ejemplo de los trapecios: si se debieran diseñar todos los cuadriláteros y después designar, la faena sería imposible. Veamos, estos y estos son los trapecios, esto no, esto sí... imposible. Por el contrario, con una definición, se aúna todo en pocas palabras.

Veamos bien cómo está hecha una definición. Mayor de edad es todo ser humano que ha cumplido 18 años. Se divide en dos partes: "mayor de edad" es el término que se debe definir, que podría ser por ahora ignorado: se llama definiendum; "todo ser humano que ha cumplido 18 años" es el predicado, tanto es designación como denominación, un conjunto de palabras aunado por la forma verbal "es", la cual sirve para definir, es decir el definiens. Ahora, si en el definiens hay palabras desconocidas, la definición sirve de poco.

Volvamos a los ejemplos matemáticos: un número primo es un número natural que tiene exactamente dos divisores; un trapecio es un cuadrilátero que tiene al menos una pareja de lados paralelos.

Primer definiendum: número primo; primer definiens: número natural que tiene exactamente dos divisores; si quien la lee no sabe qué es un número natural, o qué significa divisor, entonces no puede entender la definición. Es decir: si la designación no designa, es inútil. Segundo definiendum: trapecio; segundo definiens: cuadrilátero que tiene al menos una pareja de lados paralelos; si quien lee no sabe qué es un cuadrilátero, o desconoce el significado de lados, o de paralelos, entonces no puede entender la definición.

¿Entonces? Entonces deben existir definiciones precedentes que definan cada uno de los términos de los cuales se hace uso en el definiens: en el primer caso, definición de número natural, definición de divisor; en el segundo caso, definición de cuadrilátero, de lado, de lados paralelos. Limitémonos al segundo caso, porque en el primero nos encontramos inmediatamente en aprietos con la definición de número natural que tiene una larga y batallada historia en el panorama de la matemática. Entonces, cuadrilátero, que hace parte del definiens, ahora se convierte en definiendum; pues bien: cuadrilátero es un polígono que tiene 4 lados. Estamos como al inicio: quien lee debe saber qué significan las palabras que aparecen en el definiens: polígono, cuatro, lados. Bien, remediamos 
inmediatamente: polígono es una figura plana que tiene como contorno una línea poligonal cerrada simple.

Este proceso no puede continuar al infinito, como se podría pensar en un primer momento, simplemente porque el número de palabras a disposición en un vocabulario de cualquier idioma, es decir el número de designaciones posibles, por cuanto amplio, es finito; por tanto, a un cierto punto, el proceso se debe detener y el autor, el creador de teorías, el matemático, debe decidir por cuáles iniciar, asumiéndolas, aceptándolas sin definición.

Estas palabras se llaman "términos primitivos". Son designaciones puras, ligadas a la experiencia concreta, como afirma Stuart Mill. Ahora bien, surgen varias cuestiones vinculadas con este hecho, con estas elecciones, con los términos primitivos, posiciones epistemológicas diversas: estas palabras se asumen como términos primitivos porque son tan simples e "intuitivas" que no vale la pena definirlas, son designaciones espontáneas, basadas en culturas precedentes, se aceptan sin definición porque es su presencia en las circunstancias descritas por los axiomas las que determinan su significado; se introducen como términos puros y después la teoría que se va desarrollando les da acepciones de uso y/o definiciones implícitas.

Las soluciones filosóficas a este propósito son tantas, como también la elección de los términos primitivos es variable; por ejemplo, la palabra "punto" parece que fue elegida por Euclides como término primitivo por excelencia (pero habría tanto que decir: ¿ønukío era en verdad "punto" o era "signo"?). Dado que muchos creen, ingenuamente, que en matemática todo se define [y, aún más ingenuo: que todo se demuestra, pero sobre esto hablaremos más adelante], todo lo que hasta este momento hemos hablado sobre la definición podría ser un duro golpe a la credulidad popular. Aclaramos que hacemos referencia sólo a la definición en un puro sentido aristotélico (por género próximo y por diferencia específica), característica de la llamada "matemática elemental"; y ni siquiera tomamos en examen las definiciones por abstracción que también forman parte del vasto patrimonio de la matemática.

La definición tiene una larga historia al interior de la epistemología matemática; pero también cada una de las definiciones evoluciona, como por ejemplo aquella de ángulo (D’Amore, 1985) y aquella de 
conjunto infinito (Arrigo, D'Amore \& Sbaragli, 2010). En el aprendizaje, la sofisticada idea de definición tiene un largo tiempo de maduración; nuestra experiencia de investigación muestra cómo, generalmente, ni siquiera un estudiante con estudios profundos sabe distinguir entre denominaciones, descripciones y definiciones. $\mathrm{Y}$ muchos docentes no dominan el sentido del "género próximo" en la definición en sentido aristotélico; nos proponen, por ejemplo: cuadrado es una figura que... al puesto de: cuadrado es un rombo que...

\section{Nota}

Designación, denotación, denominación, descripción, giran alrededor del complejo y controvertido mundo de las definiciones, connotándolo de forma altamente problemática. Esto nos permitió mostrar una visión pragmatista, no realista, de la definición en matemática que trae señales de una posición filosófica oportuna.

\section{Demostración}

Para muchas personas la matemática es una disciplina de certezas, mientras que la filosofía es una disciplina de posibilidades; por lo tanto, la matemática se ocupa de la verdad, la filosofía de las posibles verdades. La pregunta banal que se desprende es: ¿qué debemos entender con esta "verdad"? Lo que asegura la verdad de la aserción matemática se llama "demostración". Veamos un ejemplo que discutiremos; no es tanto el ejemplo en sí lo que se debe examinar, sino lo que diremos al respecto más adelante.

Consideremos un triángulo $\mathrm{ABC}$ y sus ángulos internos $a, b$ y $c$

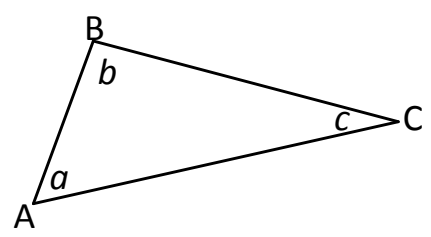

Por un objetivo cualquiera, o incluso por curiosidad, nos pedimos: ¿Cuánto mide la suma de los ángulos internos del triángulo ABC? De forma impropia pero difundida, indicamos dicha suma de amplitudes con: $a+b+c$. 
Se pueden medir las tres amplitudes con un transportador y después sumarlas como lo haría, ingenuamente, un niño; pero nosotros sabemos que en la misma acción de medir hay un error intrínseco que invalida, desde un punto de vista matemático, el resultado: hacerlo sería confundir la ingeniería con la matemática, la verdad sensible (dóxa) con la verdad de la razón (alétheia), lo concreto con el concepto, lo empírico con lo abstracto.

¿Entonces? Entonces debemos "demostrar" y no medir, es decir hacer un razonamiento convincente que no dependa de un triángulo en particular, de la situación particular, de las medidas, sino que sea general.

Un ejemplo de demostración es el siguiente: tomemos el diseño anterior; este no es el triángulo, es sólo una representación semiótica que nos ayuda en el seguimiento del razonamiento. Denotamos el triángulo abstracto con las tres letras $\mathrm{ABC}$, lo representamos semióticamente en el registro figural, trabajamos sobre la representación, pero siempre haciendo referencia al objeto triángulo de la geometría, abstracto, aquel denotado por las tres letras.

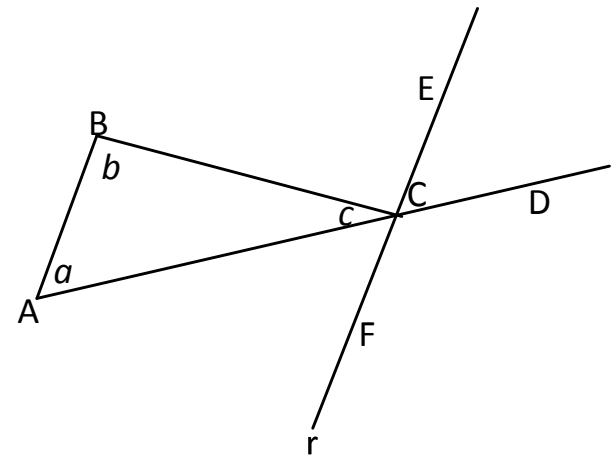

Prolongamos el lado AC más allá de $\mathrm{C}$, hasta un punto $\mathrm{D}$ elegido a placer; consideramos la recta $r$ que pasa por $C$ paralela a $A B$. Sobre ésta tomamos dos puntos $\mathrm{E}$ y $\mathrm{F}$, en semirrectas opuestas respecto a $\mathrm{C}$, que nos permitan designar los ángulos de forma oportuna. Pues bien, el ángulo BCE es igual (superponible, congruente, que tiene la misma medida... se puede decir de tantas formas) al ángulo $b$ por un teorema ya demostrado precedentemente (no en este artículo, aunque lo explica en el párrafo siguiente), en cuanto se trata de ángulos internos, es decir, formados por dos paralelas ( $\mathrm{AB}$ y $r$ ) cortadas por una transversal BC. 
$\mathrm{El}$ ángulo DCE es igual al ángulo $a$ porque son ángulos correspondientes en una situación análoga.

Pero la suma de $c$, más BCE, más DCE, forma el ángulo ACD que es un ángulo llano; por tanto la suma de los ángulos internos del triángulo $\mathrm{ABC}$ es un ángulo llano (algunos llaman la mitad de un ángulo giro, otros dicen $180^{\circ}$, otros dicen dos ángulos rectos). Y la demostración está terminada. Pero... Pero existen varias cuestiones sutiles de carácter filosófico, semiótico y lingüístico escondidas en este razonamiento.

\section{La primera}

Hicimos referencia a "teoremas precedentes", los cuales hacen a su vez referencia a teoremas precedentes, y así sucesivamente. Pero, es obvio, que este ir hacia atrás no puede ser infinito. Antes o después, para poder iniciar, se tendrán afirmaciones que deben ser tomadas como verdaderas, sin demostración, como base de todo el proceso. De esto se dieron cuenta los grandes matemáticos de la antigua Grecia, era demasiado evidente. Así que se decidió iniciar de algunas afirmaciones que se consideran verdaderas sin demostración. Por ejemplo, a un cierto punto prolongamos el segmento AC hasta un punto $\mathrm{D}$; entonces debemos dar una demostración precedente del tipo: dado un segmento, es siempre posible prolongar este segmento en cada uno de sus dos extremos.

Pero, de hecho, este es un ejemplo de afirmación que se considera verdadera sin una demostración precedente. Se llama axioma (en verdad, los matemáticos griegos distinguieron dos categorías de afirmaciones verdaderas sin demostración, los postulados y los axiomas, pero sobre este argumentos solicitamos el permiso para eludirlo).

Por tanto, contrariamente a lo que la gente cree, en matemática no es posible demostrar todo, por el contrario, se deben aceptar como verdaderas algunas afirmaciones sin demostrarlas. Lo que es filosóficamente relevante: ¿Qué es lo que las hace verdaderas? ¿Por qué precisamente éstas? ¿Cuál es la evidencia? ¿Será la adherencia a la realidad? Pero cómo... si habíamos insistido tanto en la diferencia entre doxa y alétheia...

\section{La segunda}

Hemos demostrado que en un cierto triángulo $\mathrm{ABC}$, del cual hicimos una bella figura líneas arriba, la suma de las amplitudes de sus ángulos es 
igual a $180^{\circ}$. Bien. Ahora tomemos otro triángulo y hagamos la misma demostración para ver cuánto mide la suma de las amplitudes de los ángulos internos. Y después tomamos otro y después otro y aún otro más y así sucesivamente. Cuando hayamos agotado todos los triángulos a disposición, entonces... se percibe muy bien la absurdidad de esta situación: hacer pruebas sobre todos los triángulos es imposible.

Pero, pensando un poco, nos damos cuenta de que en la demostración precedente nunca tomamos en consideración las propiedades de dicho triángulo $\mathrm{ABC}$ el cual diseñamos y designamos; por el contrario, hicimos un razonamiento general sobre un triángulo cualquiera, no sobre un triángulo en particular, sino sobre la idea misma de triángulo, sobre el objeto matemático triángulo.

Es esto lo que hace interesante, relevante y definitiva la demostración. Ahora nosotros, después de esta demostración, sabemos que en todos los triángulos del mundo (en la geometría euclídea, como veremos) la suma de los ángulos internos tiene una medida de $180^{\circ}$, no importa ni el tipo de triángulo ni sus dimensiones.

Una breve disertación didáctica: los niños de primaria a veces piensan que entre más largos sean los lados del triángulo, mayor es la suma de los ángulos internos. Para convencer a los niños pequeños, la demostración precedente es desmedida, es posible que no puedan entender ni siquiera el sentido de nuestra propuesta, por lo cual se puede recurrir a procedimientos empíricos; se diseña un triángulo en cartón o cartulina, se cortan las "puntas" del triángulo diseñado, y se reacomodan de forma oportuna para mostrar que los lados extremos están sobre la misma recta, es decir que los tres ángulos forman un ángulo llano. Para un niño de primaria el experimento concreto es más conveniente. Pero, evidentemente, es un proceso empírico, no es una demostración. Es decir, matemáticamente es irrelevante. Pero su dosis de convencimiento es suficiente para dicho nivel escolar.

Volvamos entonces al inicio. Si en matemática una afirmación es demostrada, es absurdo, inútil, es una pérdida de tiempo, intentar demostrar lo contrario. Por ejemplo: no existen ecuaciones algebraicas que tengan como raíz $\pi$. Este hecho ya se demostró. Sin embargo existen personas que buscan aún una ecuación de este tipo. Tiempo perdido. Otro ejemplo: el círculo no se puede cuadrar con regla y compás. Este hecho ya fue demostrado. Sin embargo existen personas que aún buscan esta construcción. Inútil comentar. 


\section{La tercera}

Como hemos visto en esta misma demostración, hemos nominado, después designado, después representado semióticamente, después hicimos acciones (dibujos) sobre la imagen concreta, éstas nos permitieron describir, estas descripciones nos permitieron denotar, llegando a afirmaciones que no estaban hechas sobre las representaciones semióticas sino sobre el objeto matemático en sí. Este juego de reenvíos semióticos, lingüísticos, filosóficos, que damos por descontado en un realismo ingenuo, es pragmáticamente muy complejo. ¿Hasta qué punto nos servimos de la experiencia concreta en su realización?

Existe una interesante diferencia entre el matemático y el matemático diletante; ciertamente un matemático no perdería tiempo tratando de demostrar cuestiones ya demostradas, porque sabe de antemano que significa "verdad" en matemática. Bueno, "verdad", pero habíamos dicho que la verdad se basa en axiomas, por tanto, si cambian los axiomas cambia todo. Cierto. Esta afirmación lleva a más de uno a la desesperanza, menos a los matemáticos de profesión. Tratemos por tanto de entenderla bien.

Haremos un ejemplo omitiendo las figuras, para no confundir lo que líneas abajo afirmaremos con la imagen que se podría producir. Consideremos una recta $r$ y un punto $P$ fuera de ésta. Tracemos rectas que pasan por $P$ paralelas a $r$. ¿Cuántas son dichas paralelas? ¿Una, ninguna, dos, mil, infinitas? La geometría de los Elementos de Euclides, la más clásica de las clásicas, aquella que todos han estudiado en la escuela, tiene como base la primera respuesta: es única la recta que pasa por P, paralela a $r$. (Aunque sí, en realidad, la historia sería un poco más complicada de contar, dado que desde los tiempos de Euclides e incluso antes era ya en curso un debate sobre esta cuestión).

La unicidad de las paralelas caracteriza la geometría de Euclides, pero no es una verdad demostrada, se trata de un axioma. Si fuese demostrada, no habría nada que decir, la paralela es una y basta; pero si es un axioma, cada uno de nosotros lo puede cambiar como lo desee y, precisamente, sustituirlo con otros axiomas. Existe, y es lícita, la geometría que acepta como axioma aquel que afirma que el número de paralelas es cero, por lo cual la afirmación, es decir el nuevo axioma, es: dada una recta $r$ y un punto externo $\mathrm{P}$, no existen rectas paralelas a $r$ que pasen por P. Nace una geometría muy interesante, cómoda para ciertas aplicaciones, se le conoce como "geometría de Riemann" — del nombre del matemático alemán 
Bernhard Riemann-o "geometría elíptica", pero ciertamente no es este el momento de explicar el por qué.

Existe, y es lícita, la geometría en la cual las paralelas a una recta $r$ que pasan por $\mathrm{P}$ son más de una, es decir 2 [o infinitas]; nace una geometría muy interesante, usada en aplicaciones concretas, se la conoce como "geometría de Lobačevskij" — por el nombre del matemático ruso Nikolaj Ivanovich Lobačevskij- o "geometría hiperbólica". ¿Qué significa, entonces, "verdadero" en matemática? Recordemos la famosa "definición" de Bertrand Russell: «La matemática es aquella disciplina en la cual no se sabe de qué se habla ni se sabe si aquello que se dice es verdadero o falso».

En la primera parte se afirma que, en un realismo ingenuo, los objetos de la matemática no existen, por tanto, cada una de sus denotaciones es en el vacío. Pero venimos a la segunda parte. Para muchas personas, verdadero y falso son adjetivos relacionados con la realidad empírica, concreta; verdadero es aquello que encuentra una confirmación en la realidad (si se deja caer un huevo desde un cuarto piso, llegando a tierra se romperá), falso es aquello que no tiene una confirmación en la realidad (el mismo huevo, en las mismas condiciones, se eleva acelerando hacia arriba). Para la matemática estos parámetros no existen, pues nada de ésta podríamos verificar en la realidad concreta.

Tenemos una mesa de cocina. Consideremos uno de sus bordes como si fuese una recta, después tomamos un punto sobre el borde opuesto. ¿Cuántos lados paralelos podemos trazar desde dicho punto al primer borde de la mesa? La realidad empírica nos dice: uno, pero ahora sabemos que en matemática la respuesta puede ser, es, diferente. La respuesta es: depende de la geometría en la cual nos ubicamos; y además, las cosas concretas sirven muy poco para dar razón de la verdad matemática.

¿Entonces? Entonces en matemática "verdadero" no tiene el mismo significado que tiene en la realidad empírica; "verdadero" significa: demostrado a partir de axiomas elegidos, por tanto, al interno de una cierta teoría, y si se cambian los axiomas cambia todo. "Verdadero", "falso", aún siendo términos que se usan en matemática por comodidad, son, de hecho, relativos. En lugar de decir que una determinada afirmación es verdadera, se debería decir que es coherente respecto a los axiomas elegidos; el objetivo del matemático no es la verdad, como ingenuamente se podría pensar, el objetivo del matemático es la coherencia de aquello que se demuestra en la teoría en la cual se propuso. 
La suma de los ángulos internos de un triángulo mide $180^{\circ}$, lo hemos demostrado, sí, pero lo demostramos en la geometría de Euclides, tan es así que, en un cierto momento, trazamos la recta que pasa por un punto, paralela a una recta dada; el artículo determinativo la es fuertemente condicionante. $\mathrm{Si}$ se cambian los axiomas, cambia todo, tan es así que se puede [fácilmente] demostrar que en la geometría Riemann la suma de los ángulos internos de un triángulo es mayor de $180^{\circ}$, mientras que en la geometría de Lobačevskij es menor de $180^{\circ}$. Los resultados parecen contradictorios entre ellos y, por tanto, ingenuamente, surge la idea de que sólo uno de éstos pueda ser verdadero. Pero no es así; los resultados de las demostraciones, que aquí evitamos, son coherentes respecto a la teoría, respecto a los axiomas elegidos.

No hemos terminado aquí porque nada hemos dicho acerca de la "lógica" que se usa en las demostraciones; hemos dado por descontado que la lógica, al menos esta, es única, reconocida y aceptada por todos; pero no es así. Hemos visto ampliamente cómo, en el curso de una demostración, entran en juego con prepotencia hechos relacionados con designación, denotación, denominación, descripción, definición, semiótica, lingüística y filosofía. Tan es así que en culturas diversas se ponen en juego otras formas demostrativas, a veces incluso diversas entre sí (D’Amore, 2005); sin embargo, nuestra experiencia de investigación muestra que para muchos docentes $L A$ modalidad demostrativa es única y es aquella aristotélicaeuclidiana. Es así como generalmente se usan largos e inútiles intentos de tratar la lógica simbólica elemental de primer orden con estudiantes aún no cognitivamente preparados para hacer uso de este instrumento.

\section{La reflexión didáctica y los instrumentos analíticos}

El debate filosófico sobre definición y demostración siempre ha estado acérrimo y no seremos nosotros quienes vayamos a incitarlo ni a recordar las contribuciones de los antiguos y de los modernos; entre estos últimos tan sólo citamos a Giuseppe Peano (1858- 1932), David Hilbert (18621943) y Federigo Enriques (1871- 1946).

Que la definición sea en fondo una frase y la demostración un discurso, fue tema de análisis por parte de lingüistas no matemáticos; aunque no siempre se dieron cuenta de la fineza que esconden estas dos prácticas en la interpretación al interior de nuestra comunidad de matemáticos. Se ha visto claramente, pero, en el transcurso de los años, de decenios, cómo las reflexiones sobre los instrumentos, de los cuales nosotros "expertos" nos servimos mientras 
los "novicios" hacen práctica y aprenden, sean importantes para la gestión misma de los saberes, en dos direcciones: cautela en el uso y precisión en la enseñanza, instrumento para el análisis de eventuales fracasos. Ahora, muy diferente es designar con respecto al describir o al definir, otra cosa es denotar y toda otra definir; hemos visto cómo todo esto se mezcla a diversos niveles en la acción concreta del demostrar, cuando esto se vuelve objeto de comunicación, por ejemplo en el aula escolar.

Nosotros pensamos firmemente que la reflexión siempre más analítica de los componentes que entran en juego en la praxis didáctica nos ayude a entender los fenómenos de la enseñanza- aprendizaje, con sus sutiles y por lo general ocultas complicaciones. Indicar, como dice la palabra misma, puede ser hecho con el dedo índice; se observa una figura, se le "indica" y se dice: esta figura. Todo estudiante está llamado a hacerlo de forma espontánea; muchos docentes no están dispuestos a admitir dicha indicación por parte del estudiante, al puesto de otra o en cambio de otras, que se llaman denominación y designación. Pero la base lingüísticacomunicativa- filosófica es la misma; y es una base empírica, como habíamos visto. Nos referimos al objeto concreto, el cual evoca un objeto mental, con una acción concreta. Pero si lo hacemos, en verdad, se corre el riesgo de que alguien interprete todo esto con parámetros rígidos y decida que no pueden ser aceptados.

Se parece mucho al intento totalmente abstracto de usar en la práctica matemática de aula sólo conectivos lógicos, mientras el novicio, de forma totalmente análoga a lo que sucede en la práctica matemática entre expertos, tiende a usar también conectivos epistémicos. Una sabia actividad que consideramos útil en la formación inicial de los docentes de matemática es aquella de reflexión sobre todos los términos introducidos aquí.

\section{Referencias}

Arrigo, G., D'Amore, B. \& Sbaragli, S. (2010). Infinitos. Bogotá: Magisterio. BAGnI, G. T. (2006). Linguaggio, storia e didattica della matematica. Bologna: Pitágora.

Bottazzini, U., Freguglia, P. \& Toti Rigatelli, L. (1992). Fonti per la storia della matematica. Firenze: Sansoni.

Boyer, C. B. (1968). A History of Mathematics. New York: John Wiley and Sons.

Carruccio, E. (1971a). Mondi della logica. Bologna: Zanichelli. 
. (1971b). Matematiche elementari da un punto di vista superiore. Bologna: Pitagora.

Courant, R. \& Robbins, H. (1941). What is Mathematics. New York: Oxford University Press.

D'Amore, B. (1985). 'L'idea di 'angolo' nell'antichità e sua evoluzione'. La matematica, le scienze e il loro insegnamento [1, 6-18].

. (2005a). 'La argumentación matemática de jóvenes alumnos y la lógica hindú (nyaya)'. Uno [38, 83-99]. Barcelona, España.

. (2005b). 'Secondary school students' mathematical argumentation and Indian logic (nyaya)'. For the learning of mathematics [25, 2, 26-32].

. (2005c). 'L'argomentazione matematica di allievi di scuola secondaria e la logica indiana (nyaya)'. La matematica e la sua didattica [4, 481-500]. . (2006). Didáctica de la matemática. Bogotá: Magisterio. . (2010). Matematica, stupore e poesia. Firenze: Giunti.

D’amore, B. \& Fandiño, Pinilla M. I. (2009). 'La formazione degli insegnanti di matematica, problema pedagogico, didattico e culturale'. La matematica e la sua didattica [23, 3, 261-298].

D'amore, B. \& Matteuzzi, M. (1975). Dal numero alla struttura. Bologna: Zanichelli. . (1976). Gli interessi matematici. Venezia: Marsilio.

Dummett, A. A. E. (1975). ‘¿Qué es una teoría del significado?’ Valdés, L. M. (ed.) (1991). La búsqueda del significado. Madrid: Tecnos.

Giusti, E. (1999). Ipotesi sulla natura degli oggetti matematici. Torino: Bollati Boringhieri.

Godino, J. \& Batanero, C. (1994). 'Significado institucional y personal de los objetos matemáticos'. Recherches en Didactique des Mathématiques $[14,3,325-355]$.

KLINE, M. (1972). Mathematical thought from ancient to modern times. New York: Oxford University Press.

Kutschera, F. von (1979). Filosofía del lenguaje. Madrid: Gredos.

Maier, H. (1993). 'Problemi di lingua e di comunicazione durante le lezioni di matematica'. La matematica e la sua didattica [1, 69-80].

RADFORD, L. (1997). 'On psychology, historical epistemology and the teaching of mathematics: towards a socio-cultural history of mathematics'. For the Learning of mathematics [17, 1, 26-33].

Speranza, F. (1997). Scritti di Epistemologia della Matematica. Bologna: Pitagora.

Stuart Mill, J. (1843). A System of Logic. Londres: J. W. Parker. 